\title{
Public health and welfare of our children: Working to ameliorate health determinants associated with diseases.
}

Talbert PYB*

College of Nursing and Allied Health Sciences, Howard University, Washington, D.C., USA

Accepted on July 20, 2018

\section{Introduction}

What is the role of government officials to ensure the safety and increase the health and welfare of our children? Additionally, what is the best practice to reduce the health determinants associated with diseases that continue to affect children? Historically, with some notable exceptions, state and local public health officials and public health departments have worked fervently to increase the safety and ameliorate the health of the children both locally and globally. This is vital at all levels and is fundamental because it is the way to reduce health determinants among children. Having the attention and efforts focusing from every aspect is the first component to curtail this surging problem.

Research has shown that social, behavioral, and familial factors may be associated with various types of preventable diseases among children's [1]. The World Health Organization (WHO) recently reported that about 1.3 million children died in 2015, mostly from preventable or treatable diseases. Although respiratory infections have been listed as one of the main causes of death for children worldwide in 2015, influenza, digestive disorders, viral infections, obesity, depression, and type II diabetes were found to be some of the most common serious children's health conditions [2,3]. Previous research has shown that children in the United States have been diagnosed with serious acute and chronic health conditions and diseases in progressively high numbers [4]. Therefore, an examination of negative children's health outcomes in the United States may be warranted to investigate moderate to severe risk factors which may be associated with specific preventable health issues and disease prevalence rates among American Children. Mehta, Lee, and Ylitalo [5] found that children residing in both low and middle-income households are more susceptible to preventable diseases, racial and ethnic disparities when seeking quality healthcare, and higher rates of illnesses. Additionally, there may be a growing effort to explore additional factors which may also attribute to poor health among children's groups. Recent studies suggest there are currently worldwide efforts in place to develop effective health related goals to try and reduce children's disease and mortality rates [6]. To support such initiatives, current policies should address potential factors that may be associated with disease and negative health related outcomes among children worldwide. The implementation of preventive medicine practices may critical to reducing the prevalence of diseases among children and is pertinent to reducing health determinants.

\section{Reducing Social Determinants of Health}

The primary step in reducing health determinants starts with an assiduous commitment from our government to take action against health inequities - "in collaboration with civil society, United Nations and development organizations, academia, donors and the private sector - in five priority areas (as specified by the Rio Political Declaration on Social Determinants of Health): enhancing health policies and decision-making, widening participation in policy-making and implementation, improving health care and services, strengthening international cooperation, and monitoring impact and progress" (WHO, 2018, para.1). Next, it is also imperative as the government work to restructure the Patient Protection and Affordable Care Act (PPACA) have a clear understanding of how any change can affect the health and welfare of our children, the future generation. Children need to have affordable and accessible health care insurance, and without these options, this population becomes more vulnerable to social and health determinants. Last, WHO presented an arduous global plan of action on social determinants of health that included: 1) Adopt improved governance for health and development; 2) Promote participation in policy-making and implementation; 3) Further reorient the health sector towards promoting health and reducing health inequities; 4) Strengthen global governance and collaboration; and 5) Monitor progress and increase accountability (WHO, 2018). Drawing more attention to this plan and working incongruent with the philosophy will make a difference in the lives of children worldwide.

\section{Future Scope to live for Our Children}

To reduce health determinants and disease prevalence rates to improve the health status among children, health educational initiatives that address the importance of gender equity, health care quality, economic development, and racial injustices, may assist in the establishment of appropriate health behavioral practices and the elimination of health disparities in children in America and around the world. American health organizations and agencies should continue efforts to establish and promote effective health related policies which may generate strong outreach initiatives within children's educational and recreational environments. The development of cultural awareness initiatives may also serve to generate dialogue and understanding among target populations which show significantly moderate to high rates of economic, racial and gender forms of health related disparities. The primary goal of this society should change quickly to focus on the youth and younger population. These children are the next generation, and therefore, future consideration is needed to concentrate on the health and wellness of the upcoming generation keeping in mind that the younger population is the most vulnerable segment of our society. In order to keep our children safe, leaders of today 
Citation: Talbert PYB. Public health and welfare of our children: Working to ameliorate health determinants associated with diseases. J Child Adolesc Health. 2018; 2(2):7-29.

must be passionate champions of policies and laws that help children to grow in healthier environments - at school, at home and have adequate resources to prepare for a bright future.

\section{References}

1. Yu CJ, Du JC, Chiou HC, et al. sugar-sweetened beverage consumption is adversely associated with childhood attention deficit/hyperactivity disorder. Int J Environ Res Public Health. 2016; 13(7):678.

2. Clarke C. Healthy Kids: Top 10 childhood illnesses. The West Australian. West Australian Newspapers Limited. 2014.
3. Social determinants of health. (2012) World Health Organization.

4. Compas BE, Jaser SS, Dunn MJ, et al. Coping with chronic illness in childhood and adolescence. Annu Rev Clin Psycho. 2012; 8:455-80.

5. Mehta NK, Lee H, Ylitalo KR. Child health in the United States: Recent trends in racial/ethnic disparities. Soc Sci Med.2013; 95:6-15.

6. Cha S. The impact of the worldwide millennium development goals campaign on maternal and under-five child mortality reduction: 'Where did the worldwide campaign work most effectively?'. Global Health Action. 2017; 10(1):1267961.

\section{*Correspondence to:}

Patricia Y. B. Talbert

College of Nursing and Allied Health Sciences,

Howard University,

Washington, D.C., USA

Tel: 202-806-7614

E-mail: patricia.talbert@Howard.edu 